\title{
The Kinematics Analysis of WangGuangfu 's Backward Slide Shot Put Technique
}

\author{
Jianhong $\mathrm{Xu}^{1}$, Jihe $\mathrm{Zhou}^{2}$ \\ ${ }^{1}$ Chengdu Sport Institute China 610041 \\ ${ }^{2}$ Chengdu Sport Institute China 610041 \\ 1903409781@qq.com \\ 2jihezhou123@163.com
}

Key words: shot put;back slide; kinematic analysis

\begin{abstract}
In this paper, the shot putter Wang Guangfu as the research object, and analyzes the three-dimensional video analysis of the back-stepping shot put technique of Chengdu Railway Station in the National Athletics Throw Series in 2017. The research shows that Wang Guangfu lifted the upper body prematurely during the gliding stage and the angle of the pedaling corner was too large when the right foot was off the ground. In the transitional stage, there was no good movement beyond the apparatus and no good foundation for the final exertion stage ; The last force phase, shot slower, shot angle is too large, the stage longer.
\end{abstract}

\section{Introduction}

Shot putting is a field match that is highly integrated in speed, strength, explosive force and special technology. Shot put technology has experienced the stage of putting shot in the field, the stage of shooting shot in side slide, the stage of sliding in half back, and the straight back Slide stage, slide back and slide the ball back to keep pace with such a technology development process. This paper analyzes the three-dimensional video analysis of the backward sliding technique of the first Chinese male shot putter in the track and field throwing series in 2017, points out the advantages and disadvantages, and provides a theoretical basis for the athletes to formulate the training plan in the future training and further improve the sports Score.

\section{Research purposes}

This article analyzes the three-dimensional video analysis of the backward sliding-step technology of Wang Guangfu in Chengdu Railway Station in China's track and field throwing series in 2017. Analyzes the kinematics parameters related to his skidding stage, transitional stage and final exertion stage to find out the advantages and disadvantages and gives the corresponding countermeasures and suggestions so as to further enhance the athletic performance of the athlete.

\section{Research object and method}

3.1 Research object.Wang Guangfu, male, Shot putter of Henan Province, is the first place in this competition and analytical score is 19.23 meters.

3.2 Research methods. Two Nissan JVC9800 high-speed cameras ( 50 frames/second) were used to simultaneously shoot the national track and field throwing event of 2017 . The two cameras were placed on the back and right side of the throwing circle respectively. The angle between the main axes of the two cameras was $90^{\circ}$, shooting frequency $50 \mathrm{~Hz}$. The use of satellite Titanium 3-D Signal TEC V1.0C three-dimensional video analysis software to analyze the technical action, the selection of human body model Matsui show governance ( 21 joints, 16 links, in addition to the need to add four new test points), the original data The low-pass filter smoothing, truncation frequency of $8 \mathrm{~Hz}$.

\section{Research result}

Wang Guangfu used back-sliding shot-putting technique, in order to facilitate the research and analysis, Wang Guangfu's back-to-back slide shot technique is divided into four moments and three stages. 


\section{Time division:}

T1: right foot off the ground moment;

T2: Right foot on the ground;

T3: left foot ground moment;

T4: Shot to leave the moment.

\section{Stage division:}

T1-T2: sliding stage;

T2-T3: transitional stage;

T3-T4: final exertion stage.

\section{Related parameters definition:}

Angle between the shoulder and hip axis: the angle between the horizontal axis of the shoulder and the horizontal axis of the projection of the hip in the horizontal plane.

Shot angle: shot shot speed direction and the angle formed by the ground.

Shot height: Shot in the last shot at the moment the wrist from the ground vertical height.

Stir angle: knee and ankle joints formed by the angle between the ground.

4.1 Glide stage analysis. The glide phase is from right moment (T1) to right moment (T2). From the hips active backward, and then put the left leg after the initiative, take full advantage of the "shift, pendulum," the power of the start, it will not only ensure the shot and body center of gravity to obtain the necessary speed, but also reduce the burden on the right leg, is conducive to Right leg to complete the follow-up action, and finally by stretching his right leg, recovery of the right foot to complete the slide action [1]. The main task of the gliding stage is to make the body and shot put a certain level of speed and get out of the quiescent state, paving the way for the transitional phase and the final stage of force.

Wang Guangfu in the sliding process, the maximum speed of shot is $2.65 \mathrm{~m} / \mathrm{s}$, the maximum center of gravity is $2.21 \mathrm{~m} / \mathrm{s}$, the difference between the two is $0.44 \mathrm{~m} / \mathrm{s}$ (elite athletes $0.09 \mathrm{~m} / \mathrm{s}$ ), the difference is larger, It is because of his gliding stage premature lifting of the upper body, reducing the power of the shot put distance. In the meantime, with the right foot off the ground, Wang Guangfu's right footrest (the angle formed by the line connecting the knee and the ankle to the ground) is $69.2^{\circ}$ (the world's best shot putter Andre is $54.5^{\circ}$ ), the difference is bigger, Kick the corner over the General Assembly led to the slide in the human body in the vertical direction of the speed increase, thereby reducing the level of the speed of the shot to accelerate the effect. The whole slide stage with $0.12 \mathrm{~s}$ more reasonable, indicating that he is swinging in the sliding process leg and leg support stretch active, with coordination.

4.2 Analysis of the transitional phase.The transitional phase is the time from right footed ground (T2) to left ground ground (T3). At this stage, the right foot landed in the process of falling to the left foot, the right foot buckle buckle, the center of gravity should be placed on the right leg, the body center of gravity speed should be stable and reduce the vertical displacement of the distance, the right hip to actively drive the knee joint pedal stretching and braking in time. This stage as fast and fluent as possible, the time should not be too long ${ }^{[2]}$. The main task of this stage is to form the best force posture (that is, beyond the movement of equipment), as much as possible to maintain the speed of sliding into the final stage of force, played a role in supporting.

Wang Guangfu's shoulder-hip angle (the horizontal angle between the shoulder horizontal axis and the horizontal axis of the hip) is $68.8^{\circ}$ (excellent athlete is $90^{\circ}$ ) when the left foot touches the ground. And when the left foot is touched, the distance between the projection point of the shot and the left fulcrum is $1.09 \mathrm{~m}$ (excellent athlete is $1.19 \mathrm{~m}$ ). The larger the angle of the hip axis, the more fully the body twists, and the stronger the ability to surpass the device. In contrast, Wang Guangfu did not form a good move beyond the device. Wang Guangfu's total public time in the transitional period was $0.12 \mathrm{~s}$, which was similar to that of the elite athletes in the world $(0.1 \mathrm{~s})$, indicating that the rapid kicking was accomplished during this period and the shot loss of horizontal shots was reduced. 
4.3 The last period of force analysis. The final stage of effort is from the left foot ground (T3) to the shot last shot (T4) end. The main task of this stage is to make the shot put the appropriate shot speed, shot height and shot angle. How to make good use of the final acceleration force on the shot, positive leg stretch right leg at the last force, left leg pedaling brakes and human body slide to obtain the momentum to the torso, to improve the performance of sports is of crucial importance [3].

Wang Guangfu in the last stage of shot put shot speed is $12.94 \mathrm{~m} / \mathrm{s}$, while the world's best athlete's shot speed is $13.26 \mathrm{~m} / \mathrm{s}$, compared with shot speed there is a certain gap. Second, Wang Guangfu shot in the final stage of the moment height is $2.05 \mathrm{~m}$, the world's elite shot height of about $2.2 \mathrm{~m}$, although shot height and height and arm length, reflects the physical condition of our athletes and foreign athletes gap. Wang Guangfu point shot shot in the final stage of force point of view is $44.2^{\circ}$, and in theory the shot best shot angle of $38^{\circ}-41^{\circ}$, indicating his shot angle is not reasonable. Finally, during the final hard stage, Wang Guangfu spent $0.34 \mathrm{~s}$ on the stage, $0.22 \mathrm{~s}$ higher than elite athletes. Using it for a long time would affect the transfer of the lead shot, resulting in a decrease in shot speed and an impact on the throwing result.

\section{Analysis conclusion and suggestion}

5.1 Analysis conclusion. In the gliding stage: the difference between the maximum speed of the shot and the maximum speed of the body center of gravity is $0.44 \mathrm{~m} / \mathrm{s}$, lifting the upper body prematurely and reducing the distance between the force and the shot. At the same time, the right foot off the ground when the pedal angle is too large, causing the body to increase the speed in the vertical direction. With a total time of $0.12 \mathrm{~s}$ with a more reasonable stage, swing leg and leg support stretch active, with coordination.

In the transitional phase: Wang Guangfu's shot projection distance from the left fulcrum was $1.09 \mathrm{~m}$ and the hip axis included angle was $68.8^{\circ}$, which did not result in a good move beyond the instrument and could not lay a good foundation for the final stage of force. The transitional time is $0.12 \mathrm{~s}$, which is similar to that of the elite athletes in the world, indicating that the task of rapid kicking is completed at this stage, reducing the penalty of horizontal shot speed.

Shot speed at the moment of the last shot is $12.94 \mathrm{~m} / \mathrm{s}$, the speed is slower, the height of $2.05 \mathrm{~m}$, shot angle of $44.2^{\circ}$, the angle is too large, the stage $0.34 \mathrm{~s}$, a longer time, resulting in shot speed decreased, the impact throw the grade.

5.2 suggestion. In the slide stage;Grasp the stage of attention to control the speed of body center of gravity and shot speed, make full use of the speed of the body center of gravity, the two as much as possible to maintain consistency, increase the strength of the distance from the shot put effort to reduce the pedal angle, targeted perfection slip Step Technology.

In the transitional phase;Wang Guangfu beyond the action to complete the device is not ideal, the future training, the shoulder flexibility of the training and rapid strength training and special technical training combined.

The last hard stage; In the future training process, Wang Guangfu should strengthen the training of strength, especially muscle power training, improve shot lead speed, reduce the lead shot angle and shorten the time-consuming stage.

\section{References}

[1] Wen Chao. Advanced course of track and field (Third Edition) [M] Beijing: People's Sports Press, 2012: 310.

[2] DU Hui.Analysis of Kinematics Characteristics of Back-skating Technique for Male Shot Put in Gliding Step - A Case Study of Wuhan Institute of Physical Education [J]. Contemporary Sport Science and Technology, 2016,6 (01): 152

[3] Chen Qiang. Eleventh National Games women's shot put crown, Asia, third-place shot put technology three-dimensional kinematics [D]. Capital Institute of Physical Education, 2011.

[4] Lai Qicai. Zhang Jun throwing shot put kinetic analysis [D]. Chengdu Institute of Physical Education, 2012. 
[5] Yi Jianjun. Kinematics Analysis of Chinese Elite Women's Athletes' Skid Shot Put [D] .Suzhou University, 2011.

[6] Yan Hai-feng.Analysis of 3-D Kinematic Parameters of Some Excellent Male Shot Putters in the Process of Backward Sliding Shot Put [D] .University of Suzhou, 2006. 